\title{
Le Nom propre modifié et la révolution égyptienne de janvier 2011
}

\author{
EL KHAMISSY, Riham \\ Maître de conférences au département de français de la faculté des langues (Al Alsun) \\ Université de Ain Chams, Le Caire, Égypte \\ r_e100@yahoo.com
}

L'acte de nommer se réalise dans la langue et dans le discours à travers des éléments linguistiques tels que les noms propres et les noms communs dont le statut varie selon le point de vue linguistique à travers lequel ils sont appréhendés : dénomination, désignation, périphrase.

Le Nom propre ${ }^{1}$ est "une forme définie dans la langue et attribuée comme nom, dans le monde, à un individu" (Gary-Prieur, 2005 : 59). Longuement abandonné à l'anthropologie et réservé aux pratiques lexicographiques, ce parent pauvre du nom commun, jusqu'à une époque récente, a été fortement négligé, voire "repoussé dans les marges, marges de la linguistique ou marges de la catégorie du nom commun"(Gary-Prieur, 1991 a: 12). Dans les années 80 et avec la naissance de la linguistique historique et comparative "se constitue une discipline au statut limitrophe et marginal, dans laquelle l'étude des noms propres va vivre d'une vie indépendante, l'onomastique qui étudie l'origine des noms propres, noms de personnes et noms de lieux" (Molino, 1982 : 5). Dès lors, appréhendé par des approches syntaxique et sémantique, le $\mathrm{Np}$ ne cesse de regagner de l'intérêt. En outre, le domaine de la linguistique informatique s'y intéresse et propose, à la fois, des outils de repérage et de traitement automatique correspondant à des besoins scientifiques ou industriels ( $C f$. Leroy, 2004a: 1-2).

Les travaux de linguistique qui se sont réapproprié le $\mathrm{Np}$ ont tenté, dans un premier temps, de fixer les critères définitoires prototypiques du $\mathrm{Np}$ : du point de vue graphique, la majuscule ; du point de vue morphologique, le nombre (singulier vs pluriel) ; sur le plan syntaxique, l'absence de détermination (hormis l'article au pluriel dans les noms de famille (patronyme ou anthroponyme)).

La sémantique, elle, pour définir le $\mathrm{Np}$, cherche à répondre à la question suivante : Le Np a-t-il un sens ? Certains croient que le $\mathrm{Np}$ est dépourvu de sens ; d'autres voient, au contraire, qu'il s'agit d'une mine inépuisable sur le plan sémantique (voir le tableau en annexes). Pour sortir de ce dilemme "sens vs nonsens" du Np - ou même hypersémanticité du Np vs vacuité de sens -, Georges Kleiber (1981) ramène le sens du Np à la dénomination "être appelé Np". Cette équation élabore, au dire de Wilmet (1995: 5), "le noyau atomique de la signification du nom propre autour duquel gravitent (comme des électrons) une constellation de sèmes ad libitum compressible ou dilatable". La sémantique référentielle cerne de près le fonctionnement purement logique du $\mathrm{Np}$ qui estime que, abstraction faite du contenu véhiculé, le rôle du $\mathrm{Np}$ "consiste à référer à la personne qui porte ce nom dans un univers de discours donné" (Van De Velde, 2000 : 36). D'autres conceptions sémantiques s'intéressent à la catégorisation en nom commun et en nom propre plutôt qu'au sémantisme du $\mathrm{Np}$, rejoignant ainsi la plupart des grammaires. "Le nom commun associe de façon biunivoque un signifié à un signifiant. Il est arbitraire, $i$ e, conventionnel, contraignant, contingent, quoique éventuellement congruent (...). Le nom propre en regard, à conventionalité égale, est moins contraignant, moins contingent, et plus congruent que le nom commun" (Wilmet, 1995 : 8).

Quant à la pragmatique, elle renouvelle la façon d'appréhender le $\mathrm{Np}$ en linguistique : en se penchant sur l'actualisation discursive du $\mathrm{Np}$, la pragmatique le "sémantise" et en conditionne l'interprétation en l'orientant vers une direction plutôt qu'une autre : "Les productions de sens du Np peuvent être observées en discours, comme nomination en acte. Acte de parole dans lequel le locuteur exprime un point de vue sur l'être nommé, et par lequel il prend du même coup position envers d'autres locuteurs avec lesquels il entre en relation dialogique" (Siblot et Leroy, 2000 : 102). 
Or, il faut noter que, quels que soient le niveau d'analyse ou le point de vue que révèlent ces avancées définitoires, la somme des critères définitionnels, réunis ou chacun pris séparément, ne suffit ni à définir ni à délimiter strictement la catégorie du $\mathrm{Np}$ :

Le critère de la majuscule initiale conduit à retenir, parmi les 'candidats noms propres', des termes qui n'en sont pas; le critère de l'absence de traduction ne concerne pas tous les noms propres, pas plus que celui de l'absence des dictionnaires. Le critère de l'absence de détermination et de flexion sont approximatifs et souvent contredits par les faits; les critères de l'absence de sens et de l'unicité référentielle sont discutables et concernent des noms d'objets uniques qui ne sont pas pour autant des noms propres. On remarque également que des emplois spécifiques du nom propre, déterminés et produisant des effets de sens particuliers, constituent des contre-exemples récurrents (Leroy, 2004a : 24).

Ceci n'a pourtant pas empêché les linguistes de travailler à la description et à l'analyse du Np.

Un des apports majeurs des approches linguistiques du Np consiste à examiner des constructions où le Np rompt avec les critères définitoires canoniques via des réalisations diverses. C'est pour analyser ces emplois particuliers souvent décrits par Jonasson (1991 et 1994) - comme étant à cheval entre nom propre et nom commun - qu'apparaît la notion de modification du $\mathrm{Np}$ ou celle du $\mathrm{Np}$ modifié. Il s'agit d'emplois qui s'opposent, partiellement ou entièrement, selon le cas, aux emplois standards du $\mathrm{Np}$.

Nous entendons voir, via cette contribution, si la presse, à travers des emplois et réemplois particuliers des $\mathrm{Np}$, peut façonner et conditionner notre perception de l'actualité ${ }^{2}$. Notre objectif est de démontrer que les $\mathrm{Np}$ ayant subi un changement référentiel et/ou syntaxique (en l'occurrence ceux liés à la révolution égyptienne) sont dotés d'une richesse sémantique grâce à l'éventail interprétatif qu'ils déploient et au réseau sémantico-culturel qu'ils créent dans un jeu langagier de réemploi médiatique.

Nous jetterons notre dévolu sur l'étude des Np modifiés dans le contexte de la Révolution égyptienne du 25 janvier 2011. Notre corpus couvre une période de six mois (janvier-juin 2011). Il est essentiellement composé de textes médiatiques (presse française et francophone) dans leur version papier et dans leur version électronique, principalement extraits de Libération, L'Humanité, Le Figaro, Le Point, Le Nouvel Observateur, Paris-Match, Le Journal du dimanche, La tribune de Genève, La voix du people gabonais.

\section{Le Np modifié : définitions et délimitations}

Un Np est dit "modifié" ${ }^{3}$ s'il "se présente accompagné de déterminants qui lui font perdre le caractère 'unique' ou 'singulier' fréquemment assimilé à la marque spécifique qui oppose les noms propres aux noms communs", (Kleiber, 1981 : 332). Ainsi Georges Kleiber semble-t-il considérer un Np comme "modifié" du moment où le Np est déterminé. Or, Jonasson (1994 : 12) pointe que cette condition, à elle seule, n'est pas suffisante : "le fait de se combiner avec des déterminants et des compléments n'entraîne toujours pas une modification sémantique". Leroy et Muni Toke (2007 : 171) donnent une définition plus précise du Np modifié par opposition au Np typique :

Les 'noms propres modifiés' sont des noms propres qui ne se présentent ni dans leur construction habituelle, sans article, ni avec leur fonction habituelle de désignation directe et immédiate d'un référent unique, mais au contraire se construisent avec un déterminant singulier ou pluriel, et/ou des expansions diverses, peuvent aisément abandonner la position référentielle pour se trouver en position attributive et, de plus, rompent ou modifient parfois les liens qui les unissent à leur référent attitré, pour ne le désigner que partiellement ou de façon 'oblique', voire pour renvoyer à tout autre référent.

La modification du $\mathrm{Np}$ s'opère donc aux niveaux syntaxique et référentiel et entraîne, par la suite, une modification sémantique. Nous aborderons, dans ce qui suit, les différents emplois du $\mathrm{Np}$ modifié syntaxiquement et/ou référentiellement, tout en soulignant les différentes constructions qu'ils empruntent ainsi que les effets de sens qu'ils produisent dans le contexte de la Révolution égyptienne du 25 janvier 2011. 


\section{Constructions et emplois du Np modifié}

Selon le type de relation entre le porteur du Np et le référent visé, Kleiber (1991:83-84) - en renvoyant aux travaux des linguistes ${ }^{4}$ qui se sont penchés sur le Np modifié - compte cinq emplois : dénominatif, fractionné (ou de fractionnement), exemplaire, métaphorique, métonymique. Chaque emploi possède des structures morpho-syntaxiques qui lui sont propres et est doté de valeurs discursives qui varient selon le contexte. Dans les pages qui suivent, nous en examinerons à fond quelques cas parmi les plus fréquents et les plus significatifs dans notre corpus d'étude.

\subsection{Emploi syntaxiquement modifié du Np : Emploi dénominatif}

Est dit dénominatif "l'emploi du Np précédé d'un déterminant, illustré par des exemples, comme 'il y a trois Claire dans ma classe!', 'Les Dupont sont venus nous voir! ', 'Il avait ce jour-là rencontré un Guermantes !' " (Flaux, 2000 : 136). Cet emploi est caractérisé par la détermination du Np qui se construit avec un article indéfini, singulier ou pluriel, ou défini pluriel et parfois singulier, un adjectif indéfini comme "plusieurs", un adjectif numéral "deux", "cinq", etc. Dans d'autres occurrences, des adjectifs comme "certain", "dénommé", permettent de souligner "l'acte par lequel le nom est attribué à un individu. Ce sont en quelque sorte des marqueurs du prédicat de dénomination" (Gary-Prieur, 1994 : 129). Autant dire, le contenu sémantique de cette construction pourrait être glosé comme suit : "être

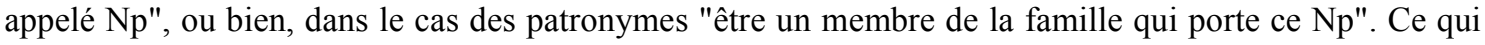
revient à pointer le caractère dénominatif du $\mathrm{Np}$.

Lorsqu'un individu est peu connu de son entourage, l'apparition du modificateur "certain" peut précéder le $\mathrm{Np}$ dénominatif pour présenter cet individu. La construction du type "un certain $+\mathrm{Np}$ " limite en quelque sorte "la connaissance que l'utilisateur de telles séquences a du référent à sa connaissance du Np de son porteur" (Schnedecker, 2005 : 100). Au dire de Riegel, Pellat et Rioul (1994:177), cette structure implique généralement "le défaut d'une connaissance plus précise du référent".

Considérons l'exemple suivant :

"On ne peut terminer (...) sans parler des «enfants de »... que sont Ali Ben Bongo, Faure Gnassingbé, Joseph Kabila, enfant-types de ce que l'on a surnommé les « monarchies républicaines » qui respectivement au Gabon, au Togo et en République démocratique du Congo n'ont eu aucun mérite pour accéder au pouvoir, si ce n'est d'avoir bénéficié de réseaux ou de systèmes dynastiques mis en place par leurs pères. Ce contre quoi se sont battus justement les manifestants de la place Tahrir, au Caire, dont le soulèvement a emporté Hosni Moubarak et fait voler en fumée le rêve de succession d'un certain Gamal... Moubarak." ("Qui, pour faire sécher les larmes des Africains? ", La tribune de Genève, 15/2/2011,

source :http://justinodaoliveirabango.blog.tdg.ch/archive/2011/02/15/qui-pour-faire-secher-leslarmes-des-africains.html, consulté le 26/6/2011).

Lorsque les rumeurs courent à propos des amendements de la Constitution en 2007, en vue de l'ascension politique de Gamal Moubarak, fils du président, et que le spectre d'une république dynastique plane à l'horizon, le pays s'embrase.L'emploi, dans l'exemple susmentionné, du patron dénominatif "un certain+ $\mathrm{Np}$ " dans "un certain Gamal... Moubarak" est très heureux, d'autant plus que le référent du Np Gamal Moubarak, avant l'an 2000, n'avait aucune activité politique, et que, même avant la révolution de janvier 2011, sa présence au Parti National démocrate (PND) soulevait des interrogations. Les points de suspension venant s'infiltrer à l'intérieur de la construction du $\mathrm{Np}$ ne sont pas anodins. Ils sont là pour confirmer l'idée de la non notoriété du fils du président il y a quelques années, ce qui implique la juxtaposition d'un patronyme d'une grande notoriété nationale et internationale ("Moubarak").

Le patron "article défini pluriel + Nom de famille" est le patron le plus représentatif de l'emploi dénominatif. Sa réalisation discursive la plus saillante est "les Moubarak" dont l'évocation est inévitable lorsqu'il est question de la corruption politico-financière, cause majeure du soulèvement des Égyptiens le 
25 janvier 2011. Il s'agit souvent de mentionner la richesse indue de la famille Moubarak et de creuser à la recherche de ses origines :

"La fortune des Moubarak dépasserait les 40 milliards" (Guillaume Errard, "La fortune des Moubarak dépasserait les 40 millards", Le Figaro, 6/2/2011)

Les Moubarak sont le président Hosni Moubarak, l'épouse influente Suzanne, l'homme d'affaires Alaa, et l'homme politique, Gamal, véritable papabile :

"La mainmise des Moubarak sur les affaires du pays ainsi que les rumeurs sur la richesse de la famille, bref, le mariage entre les entreprises et le pouvoir ont fini de discréditer le président aux yeux des Egyptiens" (propos d'Aladdin ELAASAR pour Paris-Match, "Moubarak le dernier roi d'Egypte", 12/2/2011)

Si la modification syntaxique par détermination est bien claire, au niveau référentiel, la modification est moins tangible d'autant plus que c'est bien l'individu, (ou les individus) désigné(s) par le Np "Moubarak" qui sont visés dans l'énoncé. Or, c'est parce que l'intérêt est principalement porté à leur dénomination "Moubarak" plutôt qu'à leur référent, que l'angle sous lequel ces noms propres sont considérés est déplacé et par conséquent modifié.

Assez proche de l'emploi standard en matière de référenciation, figure l'emploi exemplaire du $\mathrm{Np}$. Il s'agit, tout comme l'emploi dénominatif, d'un premier pas vers la modification.

\subsection{Emplois syntaxiquement et référentiellement modifiés du $\mathrm{Np}$}

\subsubsection{Emploi exemplaire ${ }^{5}$ du $\mathrm{Np}$}

L'emploi étiqueté "exemplaire" adopte le schème "déterminant+Np" sans expansion, c'est-à-dire ni qualification ni complément : "Le SN (...) ne contient que le Npr, en général un Npr de famille ou un Npr complet, précédé de l'article indéfini 'un' " (Jonasson, 1994 : 230). Leroy et Muni Toke (2007 : 176) ajoutent que la détermination peut se faire "soit par 'un', 'ce', soit par 'les' ". S'il est précédé de l'article défini "les", on parle de "construction phatique" réservée à l'énumération ( $C f$. Gary-Prieur, 2001 : 110115). Le $\mathrm{Np}$ exemplaire peut être le "régime de la préposition 'de' dans le complément d'un Nc (nom commun) abstrait" (Jonasson, 1994 : 230). L'emploi exemplaire a pour caractéristique principale de considérer le référent initial du $\mathrm{Np}$ comme un échantillon typique et représentatif.

Tentés par le pouvoir suprême, les dirigeants arabes sont soucieux de se coller, autant que faire se peut, aux grands héros de leur civilisation et de leur culture, ce qui les auréolerait, aux yeux de leur peuple, d'un halo sacré, les transformant en monarques quasi-divins. Ainsi lit-on dans Le Point l'interrogation suivante:

"Quel souverain arabe n'a pas frissonné en se coulant dans une grande figure du passé ou dans son effigie: un Saladin (Syrie), un Nabuchodonosor (Irak), un pharaon (Egypte) afin de paraître plus avenant aux yeux de son peuple ?" (CHEBEL, Malek,"Le monde arabe doit réussir une mutation gigantesque", Le Point, n²005, 17/2/2011)

L'évocation de Saladin et de Nabuchodonosor est une excellente incarnation de l'exemplification par recours au $\mathrm{Np}$ : les deux $\mathrm{Np}$ sont modifiés par la détermination indéfinie. Chaque $\mathrm{Np}$ est emblématique dans son pays d'origine signalé dans la glose entre parenthèses. Un Saladin pourrait être paraphrasé par "un héros et homme d'Etat syrien". Sa notoriété en tant qu'homme politique intelligent et héros de guerre à morale et aux tactiques irréprochables fait de lui une figure de proue, un idéal pour tout leader. Il en est de même pour Nabuchodonosor le souverain puissant de Babylone. Certes la notoriété de ces deux Np est une condition sine qua non pour la félicité de cet emploi du Np exemplaire, d'autant plus que les qualités des deux Np sont censées illustrer ce qui n'est pas littéralement indiqué.

La prudence excessive d'un homme politique pourrait déboucher sur l'immobilisme. Telle est l'une des causes de la chute du président Moubarak : 
"En vingt-neuf-ans de règne - le plus long qu'ait connu l'Égypte depuis le sultan Mohammed Ali-, Hosni Moubarak a toujours fait de la prudence le moteur de ses décisions. Lucide, il sait qu'il n'a ni le charisme naturel d'un Nasser, ni le bagout d'un Sadate" (GUIBAL, Claude, "Hosni Moubarak, le dictateur immobile", Libération, 12/2/2011)

Nous sommes ici face à deux occurrences typiques de l'emploi exemplaire où le Np est complément d'un $\mathrm{Nc}$ abstrait. Les $\mathrm{Np}$, Nasser et Sadate, se réfèrent aux deux prédécesseurs de Moubarak, chacun comme prototype du plus haut degré d'une qualité explicitée en toutes lettres par le co-texte proche : le charisme et le bagout. Nasser est donné ici pour le meilleur représentant de l'homme d'Etat dont le charisme et le télégénie ne font aucun doute. De même, Sadate est retenu par le discours comme emblématique de la loquacité.

Même si dans les exemples susmentionnés de l'emploi exemplaire, le référent attitré du Np coïncide avec le référent visé, cet emploi du $\mathrm{Np}$ exemplaire reste un emploi modifié : que la qualité à dimension mythique attachée par la doxa à ces $\mathrm{Np}$ soit explicitée (cas des Np Nasser ou Sadate) ou non (cas des Np Saladin ou Nabuchodonosor), le fait que le référent initial soit visé en tant que modèle ou parangon constitue, au dire de Leroy (2005 : 97), "un changement dans le mode de référence suffisant pour qu'on dépasse la simple dimension individuelle et qu'on quitte la référence standard habituellement effectuée par le Npr".

Or, il arrive souvent que le $\mathrm{Np}$ ne désigne pas entièrement son référent mais une de ses facettes. C'est d'ailleurs ce que nous allons traiter dans la rubrique qui suit.

\subsubsection{Le Np de fractionnement ${ }^{6}$}

L'emploi de fractionnement met en jeu une relation "partie-tout" entre le porteur du Np et le référent visé : "Le référent du SN avec Nom propre modifié représente, quel que soit le nom qu'on lui donne et quelles que soient les sous distinctions que l'on peut faire, seulement une 'partie' du porteur du nom" (Kleiber, 1991 : 98). Le $\mathrm{Np}$ adopte alors "une construction relativement fixe : en général, le nom propre est précédé d'un article défini suivi d'une complémentation. Celle-ci est obligatoire, puisque le nom propre ne renvoyant pas à son référent tout entier, mais à une facette de ce référent, il est nécessaire de préciser laquelle par une complémentation. Le nom propre, dans cet emploi, s'inscrit généralement dans une opposition entre deux facettes du référent (...). On peut cependant le trouver en version 'solo' avec un déterminant indéfini" (Leroy, 2004 a: 73), souvent suivi d'un adjectif qualificatif plus restrictif que descriptif, lequel ouvre un éventail d'interprétations vers un emploi modifié.

Ainsi, décrivant l'espoir de la jeunesse égyptienne dans une réforme radicale touchant tous les secteurs en Égypte, Uri Avnery écrit :

"Elles (les forces égyptiennes nouvelles) sont un rassemblement d'espoirs et d'aspirations touchant tous les domaines de la vie. Elles ont la détermination de créer 'une autre Égypte' entièrement différente de 'I'Égypte d'hier' (AVNERY, Uri, "Place Tahrir, Tel Aviv", 25/4/2011/ source : http://www.france-palestine.org/article17258.html, consulté 11/5/2011)

La formule "l'Égypte d'hier", où la complémentation (d'hier) est d'ordre temporel, laisse entendre qu'il s'agit, pour l'instance médiatique, de mettre plusieurs facettes de l'Égypte en parallèle, à savoir "l'Égypte d'hier", celle d' "aujourd'hui" et enfin celle de "demain".

L'Égypte d'hier est l'Égypte passive qui a souffert de tant de problèmes politiques, économiques et sociaux. C'est également, depuis une trentaine d'années, l'"Égypte de Moubarak"(AYAD, Christophe, " Le Caire redevient un modèle pour le monde arabe", Libération, 11/2/2011). Certes, cet emploi désigne l’Égypte à une époque bien déterminée, celle des trois dernières décennies, un pays immobile, décrit par Ayad par ces quelques mots : «L'Égypte de Moubarak est devenue, non plus un partenaire (pour les pays arabes ${ }^{7}$ ) mais un subalterne occidental au Proche-orient, voire un garde-chiourme quand il s'agit de participer au blocus de Gaza » (Ibid.). 
L'Égypte d'aujourd'hui est celle du soulèvement de la fronde qui se libère de la corruption et qui tâtonne sur la voie de la liberté. L'Égypte de demain, encore aux traits indécis, est celle qui rêve d'une vraie démocratie, de réformes, de prospérité et de gloire, celle que le Journal du Dimanche du 13 février 2011 appelle, en utilisant le Np de fractionnement : "cette Égypte du futur" :

«Maintenant, il faut rentrer chez nous, reprendre le travail pour bâtir 'cette Égypte du futur' » (Propos recueillis par MALO, Antoine, "Au Caire, le futur a déjà commencé", 13/2/2011, source : http://www.lejdd.fr/International/Afrique/Actualite/Au-Caire-le-futur-a-deja-commence268181/, consulté le 26 juin 2011)

L'Égypte du futur, c'est « une nouvelle Égypte, plurielle, pacifique et décidée » (AYAD, Christophe, " Le Caire redevient un modèle pour le monde arabe", Libération, 11/2/2011). C'est aussi celle dont parlent les manifestants sous l'appellation de "l'après-Moubarak" :

"Autour de la place Tahrir, les manifestants débattent de l'après-Moubarak et de la nouvelle Egypte à construire" (MALO, Antoine, "Au Caire, le futur a déjà commencé", 13/2/2011, source : http://www.lejdd.fr/International/Afrique/Actualite/Au-Caire-le-futur-a-deja-commence-268181/, consulté le 26 juin 2011)

Le Np "l'après-Moubarak" est ici en emploi de fractionnement et oppose cette ère nouvelle du lendemain de la Révolution à l'époque de la présidence de Moubarak. L'après-Moubarak où tout est possible, le meilleur comme le pire, où les défis sont de taille. C'est également une ère nouvelle où le mythe du président-Pharaon a sauté et où le pays est à redresser. À noter que le Np en emploi de fractionnement "L'après-Moubarak" tient lieu, en outre, de nom propre de période ou de "chrononyme ${ }^{8 "}$.

La dimension politique de la chrononymie dans "L'après-Moubarak" est indéniable vu qu'elle est construite sur la base d'un $\mathrm{Np}$ du président déchu qui a imprégné de son influence et de son autorité une époque. L'après-Moubarak restera baptisé ainsi, de façon provisoire, jusqu'à ce que se dessinent les traits de cette époque post-révolutionnaire et que l'on élise un nouveau président de la République.

Reste un dernier exemple de l'emploi du Np de fractionnement, celui de "l'Egypte de Tahrir". Construit sur le patron syntaxique "Article défini+ $\mathrm{Np}+$ Complément du Np", il a la particularité d'être composé de deux toponymes ${ }^{9}$ : "Egypte" et "Tahrir".

En nommant leur ouvrage L'Égypte de Tahrir, Claude Guibal et Tangui Salaün (2011) soulignent la nouvelle dimension que prend le Np "Tahrir". Ce Np, dont la traduction littérale en français est bel et bien "Libération", est devenu un haut lieu emblématique de ces jours de révolte qui ont jeté à bas le président Moubarak. Ainsi, "Tahrir" dépasse sa simple fonction de toponyme pour devenir un désignant événementiel (Calabrèse, 2007: 63-71), et plus précisément d'un toponyme événementiel, sorte de dédoublement lieu-événement (Leroy, $2007: 299-306$ ).

Épicentre de la Révolution, "Tahrir" devient le Np de la Révolution elle-même. Par conséquent, l'Égypte de Tahrir devient synonyme de l'Égypte de la Révolution. Aussi lit-on :

"La place Tahrir d'avant n'était pas non plus celle dont on parle aujourd'hui" (AVNERY, Uri, "Place Tahrir, Tel Aviv", 25/4/2011/ source : http://www.france-palestine.org/article17258.html, consulté 11/5/2011)

Cet emploi fractionné met l'accent sur Tahrir en tant que lieu dont le statut, avant la Révolution, est celui d'une grande place au centre du Caire. Certes, après la Révolution égyptienne du 25 janvier 2011, cette place est devenue une micro-république, autonome, libre et démocratique que l'on aimerait voir se développer à l'échelle du pays. Bien plus, le $\mathrm{Np}$ a dépassé sa référence spatiale pour devenir l'emblème d'un événement qui a bouleversé l'Egypte entière, lequel sera gravé dans sa propre Histoire.

Jusqu'ici, la modification référentielle est partielle : pour peu que l'Égypte visée soit celle de Tahrir ou du futur, le référent reste l'Égypte même s'il s'agit de l'une de ses facettes.

Or, il est des cas où la modification référentielle atteint son apogée, des cas où l'emploi modifié et l'emploi figuré s'entremêlent pour que le $\mathrm{Np}$ se donne des appréciations nouvelles qui colorent sa 
représentation. Raison pour laquelle nous porterons dans ce qui suit un regard sur l'emploi dit "métaphorique" ou "antonomasique" ${ }^{10} \mathrm{du} \mathrm{Np}$.

\subsubsection{Emploi métaphorique ou antonomasique du $\mathrm{Np}$}

Certains Np modifiés s'appuient sur une relation de similarité entre le porteur initial du Np et le référent visé par l'énoncé. Ceci n'est possible qu' "avec un Npr qui seul peut dénoter une propriété sans perdre la visée du référent, grâce au lien mémoriel qui le rattache à ce dernier" (Flaux, 1991 :35).

Ainsi, sur le plan référentiel, "le caractère métaphorique de cet emploi du Np modifié réside dans la projection sur un référent discursif ${ }^{11}$ des propriétés du référent originel" (Siblot et Leroy, 1994 : 94).

Sarah Leroy a tenu à détailler méticuleusement les critères syntaxiques et sémantico-référentiels qui permettent l'identification du $\mathrm{Np}$ en antonomase :

\footnotetext{
"Une antonomase, en discours, est un nom propre

*obligatoirement précédé d'une détermination (déterminant défini, indéfini, possessif, démonstratif, numéral) ou dans une position syntaxique qui exclut la détermination : en apposition, objet d'un verbe comme 'traiter de...', 'qualifier de...', 'traiter en...';

* optionnellement précédé (entre le déterminant et le nom propre : ce petit Mussolini) et/ou suivi (un Godard de sous-préfecture) d'un ou de plusieurs (une sorte de Boris Vian du polar) compléments (adjectifs, complément de nom, proposition relative
}

\section{ET}

* associé à un référent (personne, lieu...) qui bénéficie d'une certaine notoriété :

- notoriété 'historique', très large (Homère, Aristote, Néron, Mozart...)

- notoriété 'médiatique', plus restreinte dans l'espace et le temps (Gabin, Mandela, Bill Gates, Bernard Tapie...)

- notoriété discursive locale (le référent du nom propre a été ou sera présenté au cours du discours précédant ou suivant l'antonomase

* Ne désignant pas, ou pas seulement, le référent qui y est habituellement associé" (Leroy, 2004b : 28-29).

À noter que l'emploi antonomasique des $\mathrm{Np}$ est assez fréquent dans les textes médiatiques et prend des structures variées.

Nous examinerons d'abord les cas d'antonomases in praesentia, c'est-à-dire les antonomases "formant une expression prédicative (en particulier dans les fonctions apposition et attribut : (ex) Mathias Sindelar, le Mozart du Football, a été éconduit (Le Monde 4/12/1997) ; (ex) Dave, c'est le Marcel Proust du yéyé" (Leroy, 2003 : 168).

Dans ce type d'emploi modifié, il y a expression d'une grande ressemblance entre deux entités, ressemblance qui concerne une propriété saillante chez le porteur initial du $\mathrm{Np}$ et le référent-cible. Sur ce, nous verrons l'Égypte et sa Révolution exporter, via les textes de presse, des Np. Tel est le cas dans La voix du people gabonais, presse gabonaise et internationale, qui titre un de ces articles :

"Hillary Clinton louangeait le prochain Moubarak, Ali Bongo du Gabon" (http://www.lvdpg.com/Hillary-Clinton-louangeait-Le-prochain-Moubarak--Ali-Bongo-duGabon_a8355.html, consulté le 5 mai 2011).

L'emploi antonomasique adopte ici une structure appositive. Un Np glose un autre : Moubarak en position de support de l'apposition et Ali Bongo en position d'apport de l'apposition. Appeler Ali Bongo "le prochain Moubarak" c'est, linguistiquement parlant, exporter une ou plusieurs caractéristiques du référent du Np Moubarak sur le chef d'État gabonais, Bongo. La presse gabonaise exploite donc la notoriété de Moubarak, devenu depuis début 2011 le modèle de référence de l'homme politique corrompu et chassé par son peuple, pour pronostiquer un avenir similaire au président du Gabon. Le nom "Hosni Moubarak", 
discrédité dans le contexte politique actuel, est devenu une étiquette collée non seulement aux politiciens mystificateurs mais à tout despote quels que soient son statut, sa profession ou sa nationalité.

Des pastiches de Moubarak, il y en a aux États-Unis, notamment au Nord de Chicago. Au moment où les images des millions de manifestants à Tahrir sont projetées sur les écrans et diffusées dans les quatre coins du monde en janvier 2011, le gouverneur républicain de Wisconsin déclare son propre "état d'urgence" en annonçant qu'il s'octroie le pouvoir de licencier sans délais n'importe quel employé fédéral, et étend ce pouvoir au-delà du secteur de la fonction publique à toute la classe laborieuse, permettant à tout patron de renvoyer immédiatement les salariés récalcitrants. Conséquence : les manifestations éclatent. Les jeunes étudiants se mettent en grève par solidarité avec leurs professeurs et ne tardent pas à être secondés par les salariés le 16 février 2011.

"Ils ont convergé avec un mot d'ordre qui ne venait pas de la direction AFL-CIO mais dont la simplicité et la clarté résument l'affrontement qui se noue : Kill the Bill "tuer le décret" - ce qui veut dire, ils le savent, "tuer" aussi le gouverneur Scott Walker, surnommé Hosni Walker ou Scott Mubarak" (PRESUMEY, "Vincent, Manifestations aux Etats Unis : une place Tahrir au Wisconsin", source : http://www.unmondeagauche.fr/?page id=3383, consulté le 12/5/2011)

Les structures qu'emprunte le Npa sont en l'occurrence "Prénom1 Nom2" et "Prénom2 Nom1" où le chiffre 1 réfère au président égyptien et le chiffre 2 au gouverneur américain. Le sémantisme de ces deux constructions peut facilement être paraphrasé comme suit : Walter Scott est le Hosni Mubarak des américains. Il s'agit là d'un procédé ironique qui frise l'injure : appeler occasionnellement Scott Walter par "Hosni Walker" ou "Scott Mubarak" c'est en quelque sorte l'injurier en lui collant un Np (prénom ou patronyme) discrédité, voire disgracié par l'opinion publique et dont le référent est mal jugé par la communauté internationale.

Le Np Moubarak qui littéralement pourrait être traduit en français par le "béni" ne reçoit que malédictions. Il est même déprécié par la presse qui l'appelle désormais "honni Moubarak" (BARBANCEY, Pierre, "Égypte. Un souffle contestataire embrase tout le pays" in L'Humanité, 29 /1/2011). Ces cinq mandats successifs (de 1981 à 2011) ne lui ont valu qu'une fausse gloire qui n'a rien à voir avec la prouesse de ces prédécesseurs, ni celle des grands noms de l'Histoire égyptienne :

"Il y a des noms que tout le monde connaît et qui servent de point de repère : Ramsès II, Akhénaton, Aménophis IV. Ils sont les François Ier, Louis XIV ou Napoléon d'une immense épopée (Guibal Claude, "Hosni Moubarak, le dictateur immobile", in Libération du 12/2/2011)

La structure de l'antonomase est attributive. C'est la structure canonique de l'emploi métaphorique, qui, au dire de Nelly Flaux (1991 : 26), est "fondamentale pour le mécanisme interprétatif de l'antonomase" :

"Np1, Np2, Np3 + être + article défini + Np4, Np5, Np6 + complément déterminatif".

Les référents-cibles, (Ramsès II ("Np1"), Akhénaton ("Np2"), Aménophis IV ("Np3")) sont indiqués dans l'énoncé $^{12}$ et les Np modifiés métaphoriques (François Ier "Np4", Louis XIV "Np5", Napoléon "Np6") sont accompagnés d'un complément qui pointe le domaine dans lequel les référents-cibles endossent les caractéristiques des référents initiaux des $\mathrm{Np} 4, \mathrm{~Np} 5$ et $\mathrm{Np} 6$, à savoir être les héros de l'immense épopée politique. La journaliste de Libé, par l'emploi métaphorique des $\mathrm{Np}$ "François Ier, Louis XIV et Napoléon", importe les caractères de ces rois et empereurs qui ont marqué l'histoire de la France et fait des pharaons égyptiens Ramsès II, Akhénaton, et Aménophis ${ }^{13}$ IV des individus qui présentent avec eux des traits de ressemblance.

Pour peu que le Np Moubarak soit dénigré, celui de la place Tahrir, théâtre des affrontements et poumons de la Révolution, est honoré. C'est le lieu où les manifestants ont réussi à formuler leurs revendications et à renverser la dictature. L'esprit de Tahrir s'est propagé comme un feu de paille ranimant les assoiffés d'espoir, de bien-être, de liberté et de démocratie. Par conséquent,

"L'endroit (Tahrir) est devenu un véritable Lourdes national" (MALO, Antoine, "Au Caire, le Futur a déjà commencé", in Le Journal du Dimanche, 
http://www.lejdd.fr/International/Afrique/Actualite/Au-Caire-le-futur-a-deja-commence-268181/, 13/2/2011, consulté le 26 juin 2011).

La phrase attributive précédente est construite sur le patron suivant :

\section{Sujet + Verbe d'état + Attribut}

Le Np "Lourdes" modifié syntaxiquement par la détermination et l'adjectif antéposé est au cœur d'une construction attributive et a une fonction prédicative. Le référent-cible "L'endroit" - qui est en l'occurrence "Tahrir" - reçoit une sorte de projection de caractéristiques importées par le référent du $\mathrm{Np}$ modifié "Lourdes", Np de lieu de grande notoriété représentant une qualité qu'il appartient au récepteur de deviner en s'appuyant sur ses connaissances et sa culture. "Lourdes" est un centre de pèlerinage dans les Hautes Pyrénées. Ainsi, grâce à la foule qui afflue de tout âge et de toute classe sociale à Tahrir, la place est devenue un lieu sacré, un lieu de pèlerinage pour les manifestants qui, réprimés et oppressés, y accourent pour crier leurs revendications.

Les journalistes font de Tahrir une source d'inspiration pour les insurgés. Sur ce, plusieurs places sont dénommées, occasionnellement, "Place Tahrir".

En mars 2011, la Place de l'indépendance au Sénégal est rebaptisée la place Tahrir, en référence à la Révolution égyptienne ( $C f$. PENNETIER, Marine et FIÉVET, Jean-Loup,"Le Sénégal dénonce une tentative de coup

d'Etat, LeNouvelObservateur, 19/3/2011,http://tempsreel.nouvelobs.com/actualite/monde/20110319.REU4848/lesenegal-denonce-une-tentative-de-coup-d-etat.html, consulté le 10 mai 2011).

De même, dans Word Press, on lit le titre suivant :

"La Place de l'indépendance est à deux doigts de devenir la Place Tahrir" ("La Place de l'indépendance est à deux doigts de devenir la PlaceTahrir",19/3/2011,http://wadedegage.wordpress.com/2011/03/19/senegal-la-place-delindependance-a-deux-doigts-de-devenir-la-place-tahrir/, consulté le 5 mai 2011)

Les marches des contestataires sont annoncées contre le régime du président Abdoulaye Wade, le 19 mars, date qui rappelle son arrivée au pouvoir. Si les manifestations à Dakar n'ont pas la même ampleur que celles du Caire, la référence à Tahrir est inéluctable, un mois après la Révolution de la jeunesse égyptienne, d'autant plus que les revendications des Sénégalais rejoignent celles des Égyptiens : promotion et bien-être social, lutte pour sauvegarder les libertés individuelles. Le contexte politique est analogue : Wade est sur le seuil de briguer un troisième mandat ou de faire de son fils, Karim, un héritier politique, un successeur, c'est-à-dire un autre Gamal Moubarak.

Les airs de la Révolution traversent la méditerranée jusqu'au cœur de Madrid, à la Place Puerta del Sol. Ainsi, dans le Journal du dimanche du 20 mai 2011, Ivan Valério a choisi pour titre à son article l'interrogation suivante:

"La Puerta del Sol, nouvelle Place Tahrir ?" (Le journal du dimanche, 20/5/2011, Source : http://www.lejdd.fr/International/Europe/Actualite/Madrid-des-milliers-de-jeunes-espagnols-ontbrave-l-interdiction-de-manifester-a-la-Puerta-del-Sol-317327/, consulté le 26 juin 2011)

La structure appositive est bien claire : "La Puerta del Sol" (support de l'apposition) est appelée, via l'antonomase du Np, la "nouvelle Place Tahrir".

Survolant l'Atlantique, le Np "Tahrir" sert à désigner, à Washington, le campement prévu sur Freedom Plaza le 6 octobre 2011 (10 ${ }^{\mathrm{eme}}$ anniversaire de l'attaque américaine contre l'Afghanistan) :

"Freedom Plaza, la future Place Tahrir américaine?" (BASQUIAST, Jean-Paul, le blog du journal Média part, 8/6/2011, http://blogs.mediapart.fr/blog/jean-paul-baquiast/080611/freedom-plaza-lafuture-place-tahrir-americaine)

Même si les populations espagnole et américaine ne se battent pas contre la dictature policière, ni pour déchoir Zapatero ou Scott Walter, ils dénoncent l'abus du pouvoir, l'injustice sociale et la corruption des 
hommes politiques. Les revendications des contestataires, leur campement dans les places publiques, tout concourt pour suggérer l'image de Tahrir.

Dans certains cas, le Npa peut fonctionner in absentia, c'est-à-dire sans que le référent-cible ne soit mentionné dans le co-texte. "Ainsi, la plupart des antonomases in absentia forment des expressions référentielles et occupent dans la phrase une fonction sujet ou objet (...) (ex) Il n'y avait pas de Kenneth Starr à Pompéi (...). Au niveau microstructural, ce type d'antonomase se caractérise par une détermination indéfinie et/ou plurielle, et par l'absence ou la rareté de la complémentation" (Leroy, 2003 : 167)

Le calcul interprétatif est ici plus délicat et exige un certain effort du récepteur de l'information qui doit coopérer pour dénicher le référent-cible du Np modifié. Généralement, il s'agit d'un simple exercice pour faire sortir le lecteur de sa passivité et pour alimenter sa curiosité. Tel est bien le cas dans l'exemple suivant :

"Ils (chauffeurs en grève) viennent créer leur propre syndicat libre et interprétatif, qui réclame aussi le 'grand nettoyage' pour dégager les 'petits Moubarak' toujours à la tête de nombreuses entreprises" (Maogniss, Abdallah, "Esprit de Tahrir, es-tu encore là ?", Word press, 28/3/2011, http://egyptesolidarite.wordpress.com/2011/03/28/esprit-de-tahrir-es-tu-encore-la/, consulté le 26 juin 2011)

Certes, il est assez aisé de deviner que le référent-cible des "petits Moubarak" n'est autre que les directeurs et les chefs d'entreprises corrompus. Le $\mathrm{Np}$, via cet emploi non prototypique, semble non seulement transférer un des tares du référent original du $\mathrm{Np}$ Moubarak sur un autre référent mais semble aussi créer une catégorie ou une classe à partir du référent initial. "La dimension métaphorique intervient au niveau de la multiplication des individus de la classe ainsi constituée, qui sont tous de clones plus ou moins fidèles du référent original" (Leroy, 2003 : 169)

Il est des cas où la découverte du référent et le décodage du lien éphémère mais fort qui le lie au référent attitré sont subtils. Aucun exemple ne saurait mieux illustrer nos propos que l'exemple ci-après :

"Surtout, il faut faire disparaître toute trace de l'ancien régime, juger Hosni Moubarak et sa clique. Pratiquez une grande épuration. " Les Egyptiens ne sont pas rancuniers, il n'y aura pas de personnes tondues ni de Robespierre", tempère Nabil El Choubachy" (MALO, Antoine, "Au Caire, le futur a déjà commencé", 13/2/2011, source : http://www.lejdd.fr/International/Afrique/Actualite/Au-Caire-le-futur-a-deja-commence-268181/, consulté le 26 juin 2011)

L'énoncé de Nabil El Choubachy rapporté par Antoine Malo dans Le Journal du Dimanche, nous laisse perplexe : Robespierre est parmi les hommes politiques les plus controversés de son époque. Incorruptible révolutionnaire et grand sauveur des principes de la Révolution française de juillet 1789, il devient un dictateur en 1793-94 en exécutant les contre-révolutionnaires, en supprimant toutes les libertés et en instaurant la terreur. À notre sens, le Np "Robespierre" crée ici une catégorie, celle des bourreaux. Ceci veut dire que la Révolution égyptienne du 25 janvier 2011 ne connaîtra ni tondus ni bourreaux dans l'opération d'épuration. Autrement dit, cette révolte ne sera ni violente, ni sanguine, ni injuste, mais légale et équitable. Bref, une Révolution "blanche" et "pacifique".

Le lendemain du départ de Moubarak est marqué par les coups de balai. Si la veille, les Égyptiens étaient en liesse, ils sont conscients que l'avenir est indécis : après des décennies de répressions, les Égyptiens, ces oiseaux libérés, volent dans toutes les directions et de façon non systématique. Les militaires qui prennent en main l'avenir du pays redoutent le chaos mais "Pas tant la révolution en mode 02, selon l'expression du blogueur Wael Ghoneim, icône du soulèvement, qu'un Mai 68 version colère sur le Nil, dont tous les paramètres semblent aujourd'hui réunis". (GUIBAL, Claude, "L'armée à la manœuvre", Libération, 12/2/2011)

Le Conseil suprême des forces armées ne redoutent pas la révolution que la journaliste Claude Guibal assimile à "un Mai 68 version colère sur le Nil". L'invocation de Mai 68 français est bien visée vu que la 
jeunesse est à la base des deux soulèvements, égyptien et français. De même, la journaliste juge l'association des deux événements réussie et voit que tous les "paramètres" de Mai 68 se retrouvent dans la Révolution égyptienne du 25 janvier, sans pour autant expliciter ces paramètres en toutes lettres.

Lorsque le vent de la Révolution souffle de la Tunisie et gagne l'Égypte, nous lisons un titre, qui est, de prime abord, choquant :

"Moubarak Dehors - les "Auvergnats" entrent dans l'Histoire" (CARNEHAN, Peachy, Le mouvement international de solidarité (The international solidarity movment France), 15/2/2011, http://www.ism-france.org/analyses/MOUBARAK-DEHORS-Les-Auvergnats-entrent-dans-1histoire--article-15100, consulté le 16/5/2011).

À la lecture de ce titre, nous nous interrogeons sur ce que fait, dans le contexte de la révolution égyptienne, le Np "Auvergnat" qui renvoie aux habitants de l'Auvergne, cette province française de la Franche-Comté. L'interprétation de cette antonomase in absentia n'est pas simple pour toute personne ignorant un événement ayant eu lieu en France, il y a deux ans, événement qui porte la clé du décodage du Np modifié en question. En septembre 2009, pendant l'université d'été de l'UMP à Seignoss-Land, le ministre de l'intérieur français Brice Hortefeux a adressé des propos racistes à un jeune militant UMP, Amine Benalia-Brouch, un Auvergnat d'origine maghrébine. Depuis, il y a souvent association entre Arabe et Auvergnat. Les Auvergnats qui entrent l'Histoire, selon l'auteur de l'article, ne sont que les Arabes. Les guillemets sont un clin d'œil pour dire que le référent-cible est différent du référent conventionnel du Np Auvergnat.

Dans tous les exemples d'emploi antonomasique du $\mathrm{Np}$ que nous venons de citer, il incombe au destinataire de découvrir l'ensemble des propriétés du référent d'origine, et de trouver sous quels rapports le référent d'origine et le référent-cible peuvent être rapprochés. La maîtrise de la langue française ne suffit pas. Seul le bagage culturel du destinataire et sa connaissance du monde -et éventuellement les informations livrées par le contexte- peuvent l'aider à reconstituer le contenu associé au Np modifié.

Or, il existe des emplois du $\mathrm{Np}$ où la modification, n'est pas syntaxique, mais uniquement référentielle. C'est ce que nous envisagerons dans ce qui suit.

\subsection{Emploi référentiellement modifié du $\mathrm{Np}$ : la métaphore du $\mathrm{Np}$}

Il s'agit d'identifier un référent à un autre sans entraîner des changements de type syntaxique. Même si, sur le plan sémantique, ce phénomène donne des résultats voisins de l'emploi métaphorique du $\mathrm{Np}$, sa structure syntaxique rappelle la rigidité du Np standard.

Nous avons déjà vu comment la présence d'un déterminant devant le $\mathrm{Np}$ le rend syntaxiquement modifié et "le détourne de sa visée individualisante et en fait un classificateur" (Noailly, 2005: 40). Appliqué à un référent-cible (lors d'une construction appositive ou attributive), le Np devient également modifié sur le plan de la référence. Or, l'emploi du $\mathrm{Np}$ dans une construction le plus souvent attributive mais sans déterminants du type "SN ${ }^{14}$ est Np" ou "Np1, c'est Np2" - Np1 et Np2 étant deux Np distincts - peut être paraphrasé comme suit : Le référent de Np1 a TOUTES les caractéristiques, qualités et défauts, de Np2. Selon Flaux (2000 : 135), "la structure SN+être+attribut exprime, par elle-même, l'identification totale des deux termes mis en relation. Le référent du sujet, tout en restant lui-même, prend toutes les qualités $d u$ référent $d u \mathrm{~Np}$ attribut". Ceci pose une identité totale entre les deux $\mathrm{Np}$, certes exagérée, quant aux caractères. Flaux parle à ce sujet d'identification métaphorique $(2000: 135)$ qu'elle place aux frontières de l'antonomase alors que Leroy (2003 : 170) parle de "métaphore du Np" qu'elle définit comme étant un cas de "nom propre employé métaphoriquement sans pour autant être déterminé ${ }^{15}$ ".

La différence entre un "Np métaphorique" et une "métaphore du Np" est celle qui existe entre :

"Nous sommes tous des Khaled Saïd" (JAUVERT, Vincent, "Égypte, les faiseurs de révolution", Le Nouvel Observateur, 24-30/3/2011, p.39.) 
"Nous sommes tous Khaled Saïd" (AFP, "Mobilisation sans précédent en Egypte"), Libération, $8 / 2 / 2011)$

Dans la première occurrence, le Np "Khaled Saïd" est en emploi modifié métaphorique. Le "nous" déictique personnel ayant pour référent potentiel "les Égyptiens" est déterminé et est mis en équation avec le Np modifié ("des Khaled Saïd") et ce dans une structure attributive du type "SN+être+Np déterminé". Tous ceux qui se voient comme inclus par ce "nous" ont des propriétés communes avec Khaled Saïd : ils sont jeunes et/ou égyptiens et/ou innocents comme lui. Le portrait physique et moral de ce jeune martyr représente des lieux de convergence avec bon nombre de jeunes Égyptiens. Or, dire "Nous sommes tous Khaled Saïd", comme c'est le cas dans la seconde occurrence, c'est passer de la simple ressemblance, de près ou de loin, à l'identification, voire la superposition. Ceci témoigne de la volonté de fusionner complètement avec l'image de ce jeune homme, comme pour dire, tacitement, que toute personne aurait pu subir le même sort du jeune homme persécuté jusqu'à la mort par les policiers.

Comme une trainée de poudre, la révolution passe de la Tunisie vers l'Égypte, puis passe à la Syrie, au Yémen, à la Lybie et en Algérie. Un soulèvement du peuple arabe écrasé, muselé, réprimé, qui crie ses angoisses, qui gronde pour mettre à bas les dictatures. Dans les livres d'Histoire, les dictateurs ont des traits communs. Pour peu que la presse juge les autocrates du Maghreb arabe et du Moyen-Orient comme issus de la même estampe, les textes médiatiques ne manquent pas de souligner, via des métaphores de $\mathrm{Np}$ à la forme négative, que les pays arabes sont différents les uns des autres, et par la suite, les dirigeants des capitales arabes ne peuvent pas être considérés de la même manière. Dans ce contexte, Mireille Duteil écrit :

"Alger ne sera - pour l'heure - ni Tunis ni le Caire" (' La grande peur de Bouteflika, Le Point, $\mathrm{n}^{\circ}$ 2005, 17/2/2011, p.32.)

Elle explicite, dans son article, les raisons pour lesquelles les Algérois ne sont ni comme les Tunisois ni comme les Cairotes et explique pourquoi ils ne renversent pas leur président en dépit de leur système politique défaillant.

Le président Kadhafi qui se croit encore le leader du peuple et dont l'égocentrisme et la mégalomanie en font un tyran aux limites de la raison, s'accroche au pouvoir et ordonne à l'artillerie de viser une population qui tombe comme des mouches. Le Post du 22/3/2011 titre un de ses articles :

"Kadhafi, ce n'est pas Moubarak ou Ben Ali". (Le Post, 22/3/2011, http://www.lepost.fr/article/2011/03/22/2442718_libye-le-piegeac.html)

Dans les deux exemples précités, on dira que l'identification est modalisée par la négation. Ainsi, il est des présidents que leur peuple réussit à arracher de leur piédestal (comme Moubarak et Ben Ali), d'autres avancent des concessions et des promesses pour apaiser leur population (Bouteflika, Abdallah II, Mohamed VI), d'autres donnent la sourde oreille comme Kadhafi.

Somme toute, nous avons tenté, dans cette contribution, de mettre en relief la diversité des cas couverts par les Np modifiés dans un contexte particulier, celui de la Révolution égyptienne du 25 janvier 2011. Par ses configurations syntaxiques et ses jeux référentiels, le $\mathrm{Np}$ modifié, comme nous l'avons vu, rejoint les phénomènes de construction du sens tout en rappelant que tel ou tel dénomination occasionnelle d'une personne, d'un lieu ou d'un événement dans les textes médiatiques, n'est ni arbitraire ni objective. Nous avons tenu à montrer, pour les exemples étudiés, la motivation des emplois modifiés par rapport au référent originel ainsi que les valeurs discursives que le $\mathrm{Np}$ modifié acquiert dans son contexte de réemploi.

Que la modification soit syntaxique, référentielle, ou syntaxico-référentielle, nous avons constaté que les textes de presse centrés sur la Révolution égyptienne ne manquent pas de créativité quant à l'usage du $\mathrm{Np}$. La presse fait souvent appel au $\mathrm{Np}$ pour éveiller des images qui servent à mieux interpréter notre quotidien, en mesurant un événement ou un individu à l'aune d'un autre. Cette dénomination façonne, par sa capacité évocatrice, la manière dont le lecteur regarde l'actualité, va même, parfois, jusqu'à tester ses compétences encyclopédiques. Ces textes médiatiques convoquent donc un lecteur complice, à la fois 
actif et coopératif, prêt à se mêler au jeu d'importation/exportation qu'opère le $\mathrm{Np}$ modifié. Ce jeu consiste, pour l'instance médiatique, à faire accepter à son interlocuteur la modification suggérée du Np. $\mathrm{Au}$ dialogisme interlocutif entre l'instance émettrice et l'instance réceptrice à laquelle il appartient de trouver les points d'analogie entre le référent initial et celui visé, s'ajoute donc un brassage culturel assez fourni qui résulte de l'emploi du $\mathrm{Np}$ modifié.

Il nous semble que notre analyse a atteint, ou du moins osons-nous l'espérer, l'essentiel de l'objectif que nous nous sommes fixée: parcourir les différentes constructions des Np modifiés en nous basant sur l'exemple de la Révolution égyptienne, mettre en évidence leur fonctionnement dans ce contexte particulier, et enfin souligner la richesse sémantique qu'ils apportent grâce à l'éventail interprétatif qu'ils déploient. Nous espérons avoir prouvé, à travers cette étude, qu'appeler les choses, les êtres et les lieux par des $\mathrm{Np}$ autres que les leurs est un phénomène digne d'être étudié, tant sur le plan culturel que sur le plan langagier. 
Annexes

\begin{tabular}{|c|c|}
\hline Les Np sont vides de sens & Les Np sont riches de sens \\
\hline $\begin{array}{l}\text { Le Np "comporte une présupposition de sens, } \\
\text { dont l'accomplissement plus ou moins riche, } \\
\text { est, il est vrai, accessoire. (..) C'est justement } \\
\text { parce que le nom propre n'a pas } \\
\text { originairement de porté sémantique qu'il } \\
\text { peut d'autant plus librement s'entourer d'un } \\
\text { halo de connotations" (Granger, } 1982: 34 \text { ) }\end{array}$ & $\begin{array}{l}\text { "Les locuteurs savent (...) que les } \\
\text { anthroponymes font sens en indiquant une } \\
\text { origine géographique, ou sociale pour les } \\
\text { patronymes, une religion ou le sexe pour les } \\
\text { prénoms, ou bien encore en se chargeant de } \\
\text { savoirs liés à l'individu" (Siblot et Leroy, } \\
2000: 91 \text { ) }\end{array}$ \\
\hline $\begin{array}{l}\text { "Les noms propres n'ont pas de sens et, par } \\
\text { conséquent, la notion de signification ne } \\
\text { s'applique pas à eux" (Ullman, } 1952: 24 \text { ) }\end{array}$ & $\begin{array}{l}\text { "Si l'on classait les noms d'après la quantité } \\
\text { d'idées qu'ils éveillent, les noms propres } \\
\text { devraient être en tête, car ils sont les plus } \\
\text { significatifs de tous" (Bréal, } 1976: 183 \text { ) }\end{array}$ \\
\hline $\begin{array}{l}\text { "Les noms propres n'ont pas de sens (...) } \\
\text { Pourtant ils sont informatifs : chaque nom } \\
\text { propre drainent avec lui l'ensemble des } \\
\text { connaissances que les actants de l'énonciation } \\
\text { possèdent sur le porteur du nom. ils signifient } \\
\text { donc mais connotativement" (Kerbrat- } \\
\text { Orecchioni, 1977: 178) }\end{array}$ & $\begin{array}{l}\text { "Partant de l'aptitude du Np à signifier, nous } \\
\text { considérons ce dernier comme un outil de la } \\
\text { nomination, comme un praxème. ce concept, } \\
\text { substitué par la linguistique praxématique au } \\
\text { lexème vise une compréhension dynamique } \\
\text { des processus de réglage du sens dans } \\
\text { l'actualisation discursive, ce dont le modèle } \\
\text { clos et statique de signifié saussurien ne peut } \\
\text { rendre compte" (Siblot et Leroy, } 2000: 92 \text { ) }\end{array}$ \\
\hline $\begin{array}{l}\text { Les noms propres "n'ont pas de sens, ou un } \\
\text { type de signification unique et spécial qui les } \\
\text { distingue en tant que classe des noms } \\
\text { communs" (Lyons, 1978: 182) }\end{array}$ & \multirow[t]{2}{*}{$\begin{array}{l}\text { "Le Npr est le nom le plus significatif en } \\
\text { raison de son emploi" (Montserrat Rangel, } \\
2004: 130 \text { ) }\end{array}$} \\
\hline $\begin{array}{l}\text { "Du fait que le référent d'un nom propre est } \\
\text { normaleùent unique, on conclut parfois que le } \\
\text { nom propre est une simple étiquette colle à } \\
\text { une chose, qu'il a un référent, pas un sens" } \\
\text { (Ducrot et Todorov, } 1972: 321 \text { ) }\end{array}$ & \\
\hline
\end{tabular}

\section{Bibliographie}

\section{I- Corpus : les articles de presse cités}

AVNERY, U., "Place Tahrir, Tel Aviv", 25/4/2011/ source : http://www.france-palestine.org/article17258.html, consulté $11 / 5 / 2011$.

AYAD, Ch., "Le Caire redevient un modèle pour le monde arabe", Libération, 11/2/2011.

BARBANCEY, P., "Égypte. Un souffle contestataire embrase tout le pays" in L'Humanité, 29 /1/2011, source: http://www.humanite.fr/28_01_2011-egypte-un-souffle-contestataire-embrase-tout-le-pays-463547

BASQUIAST, J-P., " Freedom Plaza, la nouvelle place Tahrir américaine", le blog du journal Média part, 8/6/2011, http://blogs.mediapart.fr/blog/jean-paul-baquiast/080611/freedom-plaza-la-future-place-tahrir-americaine

CHEBEL, M.,"Le monde arabe doit réussir une mutation gigantesque", Le Point, n²005, 17/2/2011, p.36) 
DUTEIL, M., "La grande peur de Bouteflika", Le Point, n²005, 17/2/2011, pp.32-35.

ELAASAR, A. pour Paris-Match, "Moubarak le dernier roi d'Égypte", 12/2/2011)

ERRARD,G., "La fortune des Moubarak dépasserait les 40 millards", Le Figaro, 6/2/2011

GUIBAL C., "Hosni Moubarak, le dictateur immobile", Libération du 12/2/2011

GUIBAL, C., "L'armée à la manœuvre", Libération, 12/2/2011

JAUVERT, V., "Égypte, les faiseurs de révolution", Le Nouvel Observateur du 24-30/3/2011, pp.37-39.

MALO, A., "Au Caire, le Futur a déjà commencé", in Le Journal du Dimanche, http://www.lejdd.fr/International/Afrique/Actualite/Au-Caire-le-futur-a-deja-commence-268181/, 13/2/2011, consulté le 26 juin 2011

MAOGNISS, A., "Esprit de Tahrir, es-tu encore là ?", Word press, 28/3/2011, http://egyptesolidarite.wordpress.com/2011/03/28/esprit-de-tahrir-es-tu-encore-la/, consulté le 26 juin 2011)

PENNETIER, M. et FIÉVET, J-L.,"Le Sénégal dénonce une tentative de coup d'Etat, LeNouvelObservateur,19/3/2011,http://tempsreel.nouvelobs.com/actualite/monde/20110319.REU4848/le-senegaldenonce-une-tentative-de-coup-d-etat.html, consulté le 10 mai 2011

PRESUMEY, V., "Manifestations aux États-Unis : une place Tahrir au Wisconsin", source : http://www.unmondeagauche.fr/?page_id=3383, consulté le 12/5/2011)

VALÉRIO, I., "La Puerta del Sol, nouvelle Place Tahrir ?" (Le journal du dimanche, 20/5/2011, mise à jour 21/5/2011, http://www.lejdd.fr/International/Europe/Actualite/Madrid-des-milliers-de-jeunes-espagnols-ont-brave-1-interdiction-demanifester-a-la-Puerta-del-Sol-317327/, consulté le 26 juin 2011.

AFP, "Mobilisation sans précédent en Égypte", Libération 8/2/2011.

"Hillary Clinton louangeait le prochain Moubarak, Ali Bongo du Gabon" (http://www.lvdpg.com/Hillary-Clinton-louangeaitLe-prochain-Moubarak--Ali-Bongo-du-Gabon_a8355.html, consulté le 5 mai 20011.

"La Place de l'indépendance est à deux doigts de devenir la PlaceTahrir",19/3/2011, source $: \mathrm{http}: / /$ wadedegage.wordpress.com/2011/03/19/senegal-la-place-de-lindependance-a-deux-doigts-de-devenir-laplace-tahrir/, consulté le 5 mai 2011

"Moubarak le dernier roi d'Egypte", Paris-Match, 12/2/2011, source: http://www.parismatch.com/ActuMatch/Monde/Actu/Moubarak-le-dernier-roi-d-Egypte-250801/)

"Qui, pour faire sécher les larmes des Africains? ", La tribune de Genève, 15/2/2011, source : http://justinodaoliveirabango.blog.tdg.ch/archive/2011/02/15/qui-pour-faire-secher-les-larmes-des-africains.html, consulté le 26/6/2011).

\section{II- $\quad$ Sur le Nom propre}

a) Ouvrages

GARY-PRIEUR, M-N., (1994), Grammaire du nom propre, presses universitaires de France, Paris.

GARY-PRIEUR, M.-N., (2001), L'individu pluriel. Les Noms propres et le nombre, CNRS, Paris

JONASSON, K., (1994), Le Nom propre, Construction et Interprétation, Duculot, Louvain-la-Neuve.

KLEIBER, G., (1981) Problèmes de référence : descriptions définies et noms propres, Université de Metz, Metz.

LEROY, S., (2004a), Le Nom propre en français, éd. Ophrys, Paris.

b) Chapitres d'ouvrages collectifs

BUCHI, E., (1996), "Les structures du 'Franzoïsiche Etymologisches wöterbuch". Recherches métalexicographiques et métalexicologiques, éd. Niemeyer, Tübingen.

MONTSERRAT RANGEL, V., (2004) "Le nom propre en discours : statut et fonctionnement des informations référentielles", in La nomination : quelles problématiques, quelles orientations, quelles applications ?, ouvrage collectif sous la dir de Françoise Dufour, Elise Dutilleul-Guerroudy, Bénedicte Laurent, éd. Praxiling CNRS, Université Paul Valéry-Montpellier 3, 2004, Montpellier, pp.129-139. 
c) Articles de revues scientifiques

BACO, P., DOUZOU, L., et HONORÉ, J.-P., (2008) "Chrononymes. La politisation du temps", in Mots. Les langages du politique, n87, pp.5-12.

BALAYN, J-D. et MEYER, B. (1981), "Autour de l'antonomase du Nom propre", in, Poétique n 46, 1981, pp.183-199

CALABRÈSE, L. (2007), "L'émergence du contexte dans les désignants événémentiels : intuition du chercheur ou matérialité discursive ?", in Analyse du discours et contextes, Actes du Ve colloque jeunes chercheurs Praxiling, Montpellier 10-11 mai 2007, sous la dir de Marion Sandré, éd lambert Lucas, Limoges, pp.63-71.

FLAUX, N., (1991), "L'antonomase du nom propre ou la mémoire du référent", in Langue française, n92 (« Syntaxe et sémantique du nom propre »), pp.26-45.

FLAUX, N., (2000), "Nouvelles remarques sur l'antonomase", in Lexique $\mathrm{n}^{\circ} 15$, (« Les noms propres : nature et déterminations »), Presses universitaires du Septentrion, Lille, pp.117- 144.

GARY-PRIEUR, M.-N., (1989),"Quand le référent d'un nom propre se multiplie", in Modèles linguistiques, 11-2, 1989, pp.119-133.

GARY-PRIEUR, M-N., (2005), "Où il est montré que le nom propre n'est (presque) jamais modifié", in Langue française, $\mathrm{n}^{\circ}$ 146, (« Noms propres : la modification »), pp.53-66.

GARY-PRIEUR, M-N, (1991a), "Le nom propre constitue-t-il une catégorie linguistique ?", in Langue française, $\mathrm{n}^{\circ} 92$, ("Syntaxe et Sémantique du nom propre"), pp.4-25.

GARY-PRIEUR, M-N, (1991b)"La modalisation du Np", Langue française, n92, ("Syntaxe et Sémantique du nom propre") pp.46-63.

GRANGER, G., (1982),"À quoi servent les noms propres ?", in Langages nº 66 ("Le Nom propre"), pp.21-36

JONASSON, K., (1991), "Les noms propres métaphoriques: construction et interprétation", Langue française, $\mathrm{n}^{\circ} 92$, ("Syntaxe et Sémantique du nom propre") pp. 64-81.

KLEIBER, G., (1991), "Du nom propre non modifié au nom propre modifié : le cas de la détermination des noms propres par l'adjectif démonstratif", in Langue française, n92, ("Syntaxe et sémantique du Np »), pp.82-103.

LEROY, S. et MUNI TOKE, V. (2007), "Une date dans la description linguistique du Nom propre : L'Essai de la grammaire de la langue de Damourette et Pinchon", in Lalies, n²7, ENS, Paris, pp.115-190.

LEROY, S., (2003), "Antonomase, métaphore et nom propre : identification ou catégorisation ?", in Travaux linguistiques du CERLICO, n`16 ("Morphosynyaxe du lexique-2, Catégorisation et mise en discours »), PU de Rennes, Rennes, pp 161178.

LEROY, S., (2004 b), "Extraire sur patrons : allers et retours entre analyse linguistique et repérage automatique", in Revue Française de linguistique appliquée ${ }^{\circ} \mathrm{IX}-1$, (Linguistique et informatique : nouveaux défis), éd. De Werelt, Amsterdam, pp.25-43.

LEROY, S., (2005), "L'emploi exemplaire, un premier pas vers la métaphorisation ?", in Langue française $\mathrm{n}^{\circ} 146$, (« Noms propres : la modification »), pp.84-98.

LEROY, S., (2007), "Noms propres et Transcatégorisations. Le cas des toponymes événémentiels" in Actes du XXVe Congrès internationale de linguistique et de philologie romane, Innbruck, 3-8 septembre 2007, sous la dir. de M. Ilescu, H. SillerRunggaldier, P. Danler, éd. Niemeyer, Tübingen, pp.299-306

MOLINO, J., (1982), "Le nom propre dans la langue", in Langage, n 66, ("Le Nom propre"), juin 1982, pp.5-20

NOAILLY, M., (2005) "Être Chateaubriand ou rien", in Langue Française, n ${ }^{\circ} 46$, (« Noms propres : la modification »), pp.39-52.

SCHNEDECKER, C., (2005), "Quand 'un certain' David Bowie repoussait les limites du bon goût..." ou que modifie 'certain' dans les séquences un+certain+nom propre?", in Langue française, $\mathrm{n}^{\circ} 146$, juin 2005 , ("Les noms propres : la modification"), pp.99-113.

SIBLOT, P. et LEROY, S., (2000), " L'antonomase entre nom propre et catégorisation nominale", in Mots. Les langages du politique, $\mathrm{n}^{\circ} 63$, ENS, pp.89-104. 
VAN DE VELDE, D., (2000) "Existe-t-il des Np de temps ?", in Lexique $\mathrm{n}^{\circ} 15$, ("Les noms propres : nature et détermination"), Presses Universitaires du Septentrion, Lille, pp.35-45.

WILMET, M., (1995),"Le nom propre en linguistique et en littérature", Communication à la séance mensuelle du 13 mai 1995. Académie Royale de langue et de littérature françaises de Belgique, Bruxelles. source : www.arllfb.be, consulté le 15 mai 2010.

\section{III- Ouvrages de linguistique}

BREAL, M., (1976), Essai de sémantique, éd. Slatkine Reprints, Genève, (1 ${ }^{\text {ère }}$ éd. 1924)

KERBRAT-ORECCHIONI, C., (1977), La Connotation, Presses Universitaires de Lyon, Lyon.

LYONS, J., (1978), Éléments de sémantique, Larousse, Paris

RIEGEL, M., PELLAT, J-Ch. et RIOUL, R., (1994), Grammaire méthodique du Français. Linguistique nouvelle, PUF.

ULLMAN, S., (1952), Précis de sémantique française, éd. Francke, Berne.

\section{IV- Dictionnaire de linguistique}

DUCROT, O. et TODOROV, T., (1972), Dictionnaire encyclopédique des sciences du langage, éd. Seuil, Paris.

\section{V- Ouvrage sur la Révolution égyptienne}

GUIBAL, Claude et SALAÜN, Tangui, (2011), L'Égypte de Tahrir. L'anatomie d'une révolution, Seuil, Paris.

Notes.

${ }^{1}$ Désormais Np.

${ }^{2}$ Pour ce faire, nous envisageons de recourir aux notions développées dans le cadre théorique des Np modifiés. Les références aux travaux de Georges Kleiber, Krestin Jonasson, Marie-Noëlle Gary-Prieur, Nelly Flaux, Sarah Leroy, Michèle Noailly en témoignent.

${ }^{3}$ Cette appellation est empruntée à Burge : "L'existence théorique des 'noms propres modifiés' dans ce cadre linguistique est assez récente, (...) le terme est emprunté au philosophe Burge (1973)". LEROY, Sarah et MUNI Toke, Valelia. (2007 : 171).

${ }^{4}$ Notamment Jonasson et Gary-Prieur.

${ }^{5}$ Appelé "emploi quidditif" par Jean-Daniel Balayn et Bernard Meyer (1981 : 187).

${ }^{6}$ Cet emploi est dit "manifestatique" ou "de manifestation" selon Jonasson (1994 : 173). C'est le Np "référant (...) à différents aspects ou manifestations du particulier visé par le $N p "$. Gary-Prieur parle à ce propos de "multiplication" (1989:119-133) et de modalisation (1991b : 46-63), voire d'image (1994:36).

\footnotetext{
${ }^{7}$ Nous explicitons.
}

${ }^{8}$ Un "chrononyme" est une "expression, simple ou complexe, servant à désigner en propre une portion de temps que la communauté sociale appréhende, singularise, associe à des actes censés lui donner une cohérence, ce qui s'accompagne du besoin de la nommer (...). Sur le plan morphologique, les chrononymes les plus caractéristiques se présentent comme des noms ou des syntagmes nominaux figés (la Belle époque, les Années de plomb (...)). On ne 
saurait toutefois négliger, en raison de la grande productivité du modèle, des formations plus libres élaborées à partir du mot du lexique calendaire et dans lesquels le second terme est une variable construite parfois indirectement (le siècle de Périclès), parfois directement (les Années de Mitterrand/Giscard/Chirac/Pilote) ...) (...) D'autres dénomment le temps par ouverture ou fermeture à partir d'un événement saillant évoqué de façon directe (l'Avantguerre) ou plus détournée (L'Après-De-Gaulle)" (Baco, Douzou et Honoré, 2008 : 5).

${ }^{9}$ Les toponymes sont "des noms de lieux et comportent différentes subdivisions : noms de villes et de pays (Paris, Albanie), mais aussi noms d'unités géographiques plus petites comme les noms de quartiers (Recouvrance, Bastille), noms de montagnes (le mont Ventoux, les Alpes), de mers et de cours d'eau (l'Atlantique, la Seine, la Durance). On peut y inclure les noms de rues (rue de Rivoli), de déserts (le Sahara), d'édifices ou de monuments (la Tour Eiffel, l'Arc de Triomphe), d'installations militaires (le pentagone, la ligne Maginot) ..." (Leroy, 2004 : 34).

${ }^{10} \mathrm{Si}$ Kleiber, Jonasson et Gary-Prieur appelle cet emploi "Np modifié métaphorique" ou "emploi métaphorique du $\mathrm{Np}$ ", Flaux, Siblot et Leroy préfère s'en tenir à l'appellation d'"antonomase du Np" ou "Np antonomasique" (désormais Npa).

${ }^{11}$ À ce propos, Jonasson (1994) parle de référent discursif. Or, l'adjectif "discursif" implique un référent mentionné en discours. Sarah Leroy (2003 : 166) propose l'appellation référent-cible qui n'exige pas une présence du référent dans le co-texte.

${ }^{12}$ Puisque l'antonomase est in praesentia.

${ }^{13}$ Signalons au passage que Claude Guibal a commis une maladresse en considérant Akhénaton et Aménophis IV comme deux pharaons distincts alors qu'Akhénaton est le surnom que s'est donné Aménophis IV suite à l'adoration de son dieu unique 'Aton" qui symbolise le disque solaire.

${ }^{14}$ Le SN (Syntagme nominal) peut avoir un Np à sa tête ou peut être composé de Nc uniquement.

${ }^{15} \mathrm{Ne}$ sont pas concernés les cas d'absence d'articles devant un Np liées à la position appositive à l'application de la règle de cacophonie, le régime de la préposition, à un emploi dans une phrase nominale. Ces cas sont comme ceux où le Nc lui-même est employé sans article. Ils sont listés par Meyer et Balayn (1981 :192). 